\title{
MORE RESULTS ON THE SMALLEST ONE-REALIZATION OF A GIVEN SET II
}

\author{
Kefeng Diao, Fuliang Lu
}

\author{
AND \\ PING ZHAO ${ }^{1}$ \\ School of Mathematics and Statistics \\ Linyi University \\ Linyi, Shandong, 276005, China \\ e-mail: kfdiao@163.com \\ flianglu@163.com \\ zhaopingly@163.com
}

\begin{abstract}
Let $S$ be a finite set of positive integers. A mixed hypergraph $\mathcal{H}$ is a onerealization of $S$ if its feasible set is $S$ and each entry of its chromatic spectrum is either 0 or 1 . The minimum number of vertices, denoted by $\delta_{3}(S)$, in a 3-uniform bi-hypergraph which is a one-realization of $S$ was determined in [P. Zhao, K. Diao and F. Lu, More result on the smallest one-realization of a given set, Graphs Combin. 32 (2016) 835-850]. In this paper, we consider the minimum number of edges in a 3-uniform bi-hypergraph which already has the minimum number of vertices with respect of being a minimum bihypergraph that is one-realization of $S$. A tight lower bound on the number of edges in a 3-uniform bi-hypergraph which is a one-realization of $S$ with $\delta_{3}(S)$ vertices is given.
\end{abstract}

Keywords: mixed hypergraph, feasible set, chromatic spectrum, gap, onerealization.

2010 Mathematics Subject Classification: 05C15,05C35.

Received 22 June 2016

Revised 29 August 2017

Accepted 15 September 2017

\footnotetext{
${ }^{1}$ Corresponding author.
} 